\title{
Critical care echocardiography: a certification pathway for advanced users
}

\author{
Scott J. Millington, MD (1) - Alberto Goffi, MD • Robert T. Arntfield, MD
}

Received: 6 December 2017/Revised: 13 December 2017 / Accepted: 15 December 2017/Published online: 16 January 2018

(C) Canadian Anesthesiologists' Society 2018

The content of this issue of Journal supports the broad overall acceptance that point-of-care ultrasound (POCUS) has achieved across many medical specialties. Despite this progress, fundamental confusion persists surrounding the achievement of both competency and certification in POCUS. In this editorial, we seek to demystify and clarify these issues from a Canadian critical care perspective, with special consideration of the recently announced examination and certification pathway in advanced critical care echocardiography.

The landscape of competency and certification in POCUS cannot be understood without a firm grasp on the nomenclature that has been adopted thus far (Figure). For POCUS performed by intensivists, critical care ultrasound (CCUS) is the appropriate umbrella term, spanning the two main branches of critical care echocardiography (CCE) and general critical care ultrasound (GCCUS). ${ }^{1}$ Critical care

\section{S. J. Millington, MD ( $\varangle)$}

Critical Care Medicine, The Ottawa Hospital, The University of Ottawa, Ottawa, ON, Canada

e-mail: smillington@toh.ca

\section{A. Goffi, MD}

Interdepartmental Division of Critical Care Medicine, University of Toronto, Toronto, ON, Canada

A. Goffi, MD

Department of Medicine, Division of Respirology (Critical Care), University Health Network, Toronto, ON, Canada

\author{
A. Goffi, MD \\ Department of Medicine, University of Toronto, Toronto, ON, \\ Canada \\ R. T. Arntfield, MD \\ Division of Critical Care Medicine, London Health Sciences \\ Centre, Western University, London, ON, Canada
}

echocardiography itself is divided into basic (BCCE) and advanced (ACCE) skill sets.

A basic-level provider should be able to generate the five core transthoracic views, interpret left and right ventricular size and global function, identify a pericardial effusion, assess the inferior vena cava, and recognize catastrophic leftsided valvular pathology. So essential to the modern practice of critical care medicine, BCCE has been endorsed as a core skill for all intensivists by the worldwide critical care community ${ }^{1,2}$ and benefits from a growing evidence base. ${ }^{3}$ As with other essential intensivist skills (bronchoscopy, central venous access, airway management), BCCE (and likewise GCCUS) should be taught under local expert supervision during fellowship training. Importantly, formal certification for BCCE and GCCUS has been deemed unnecessary both internationally ${ }^{1}$ and by the unanimous consensus of Canadian CCUS experts, ${ }^{4}$ a position endorsed by the Canadian Critical Care Society in 2013.

When it comes to advanced echocardiographic applications, there is a very important departure in the approach and philosophy surrounding training and certification. Advanced applications require greater cognitive and procedural skill, are more quantitative (and therefore subject to error), and are overall closer in scope to a diagnostic echocardiographic examination performed by a cardiologist or an intraoperative examination performed by a cardiac anesthesiologist. Within the scope of ACCE, an intensivist may use a transthoracic or transesophageal approach to address hemodynamic questions (cardiac output, volume responsiveness, or heart-lung interactions), to resolve diagnostic questions (shock etiology, source of embolism, endocarditis, or important valvular dysfunction), or to guide procedures (pacemaker insertion or extracorporeal membrane oxygenation cannulation). Due to this inherent sophistication and resemblance to comprehensive echocardiography, there is 


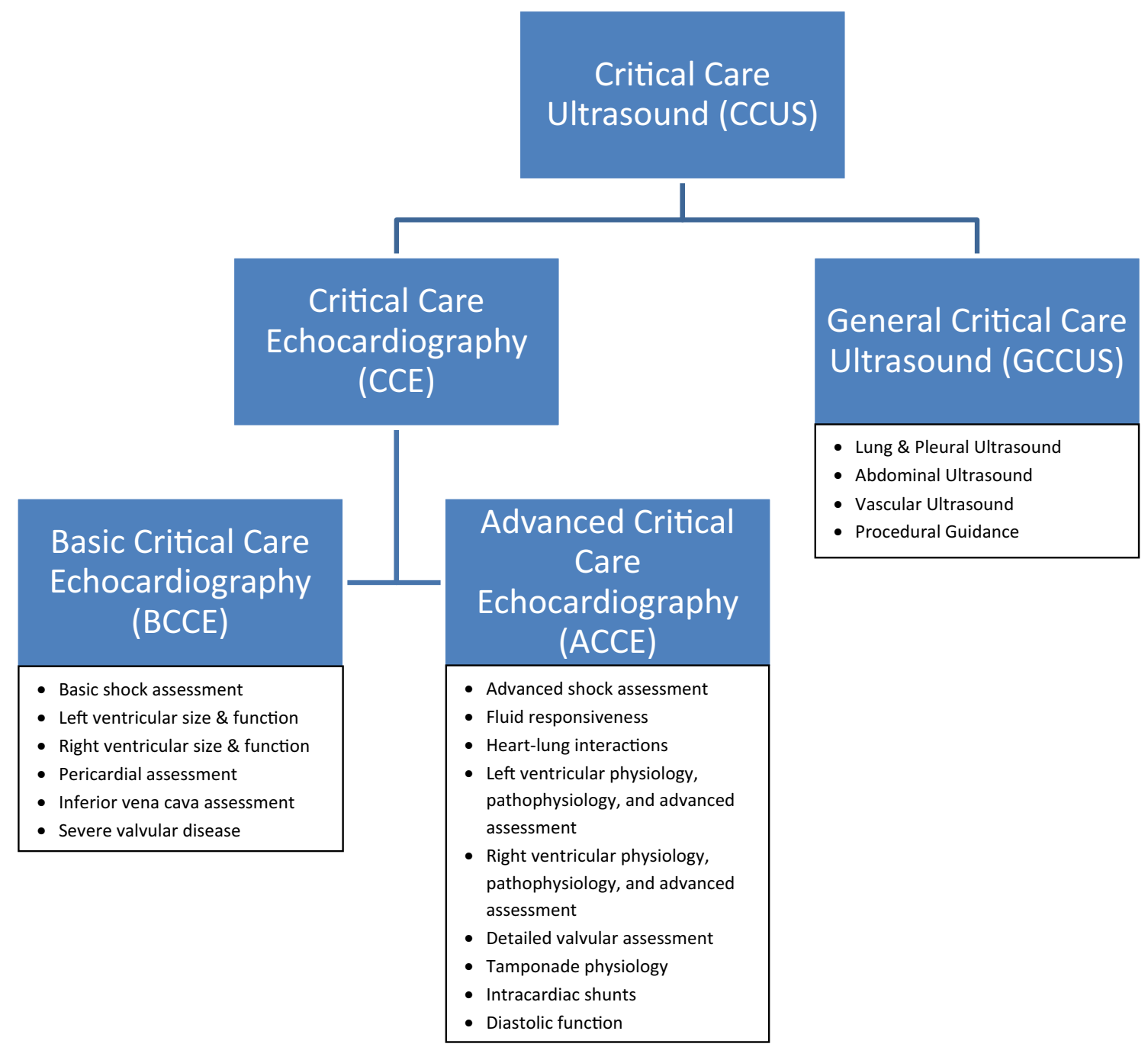

Figure Overview of terminology and scope of practice for critical care ultrasound

Vue d'ensemble de la terminologie et du champ d'application de l'échographie en soins intensifs

international agreement that formal certification is desirable. ${ }^{2,5}$ While North America has not (until now) had a mechanism to honour this decade-old call for advanced certification, other jurisdictions have had success in implementing initiatives such as the European Diploma in EchoCardiography program. ${ }^{6}$

As of 2019, however, examination and certification in advanced critical care echocardiography in North America will become a reality. The National Board of Echocardiography (NBE), the same organization that oversees certification for comprehensive and perioperative echocardiography, has partnered with multiple North American critical care societies to create an examination and certification pathway for ACCE. This process has broad support, including from The American College of Chest Physicians, The Society of Cardiovascular Anesthesiologists, The Society of Critical Care Medicine, The American Thoracic Society, The American Society of Echocardiography, and The
American College of Emergency Physicians, among others. Although American-based, NBE examinations have long been used by other specialty groups in Canada, and the value of certification will be similarly impactful with this new pathway being forged.

Though the details are still being finalized, the critical care NBE pathway will bear many similarities to the current certification process for cardiologists and cardiac anesthesiologists. In particular, the critical care track will involve a standardized examination of both knowledge (through an advanced assessment test) and practical training; applicants will need to show proof of completion of an echocardiographic portfolio, supervised locally. The goal of this certification process is to legitimize and support the use of the ACCE skill set by intensivists who have met the high standards of cognitive and procedural training outlined in the international ACCE training consensus statement. ${ }^{5}$ 
In considering the ACCE certification model, the recent historical example provided by cardiac anesthesiology may be instructive. Faced with the similar challenge of adopting an advanced ultrasound skill into routine practice, the specialty embraced a similar advanced certification pathway model. This route helped overcome the difficult transition to achieving a critical mass of competent advanced providers, analogous to the current critical care climate with ACCE. Just as certification in advanced intraoperative echocardiography was a key milestone for the specialty of cardiac anesthesiology, we anticipate that ACCE certification for intensivists will contribute to a new era in the way critical care medicine is practiced.

While interest is expected to be strong, ${ }^{7}$ it must be stressed that the new NBE pathway does not relate to or restrict the routine use of basic echocardiographic applications by those who are appropriately trained. No barriers of any kind should be erected to obstruct the use of basic ultrasound applications by critical care practitioners. Basic Critical Care Echocardiography and GCCUS are competencies of critical care medicine ${ }^{8}$ and their use should continue to flourish, guided by local experts in accordance with national ${ }^{4}$ and international ${ }^{2}$ guidelines.

We are supportive of this new certification pathway for ACCE and will encourage all burgeoning leaders in CCUS to seek certification. The NBE's history of successful administration of echocardiography examinations coupled with widespread society support will, we anticipate, lead to strong uptake and a positive reception across the North American critical care community. In addition to certifying those with competence in ACCE and officially bringing advanced echocardiography in to North American critical care practice, this new pathway will have two additional effects that will benefit critical care as a whole. First, those certified in ACCE can be relied upon to become champions at the local level, helping to meet the current shortfall of oversight and training in BCCE. Second, an unintended consequence of advanced certification is that BCCE and GCCUS, through their purposeful omission from this pathway, will be all the more recognized as core intensivist skills. As a result, educators and training regulators can and must act to ensure their greater dissemination.

\section{L'échocardiographie aux soins intensifs: une voie vers la certification pour les utilisateurs avancés}

Le contenu de ce numéro du Journal soutient l'acceptation généralisée obtenue par l'échographie au point d'intervention (PoCUS) dans de nombreuses spécialités médicales. En dépit de ce progrès, il persiste une confusion fondamentale autour de l'obtention, à la fois, de la compétence et de la certification en matière d'échographie PoCUS. Dans cet éditorial, nous cherchons à démystifier et clarifier ces questions du point de vue des soins intensifs au Canada, en nous intéressant plus particulièrement aux parcours récemment annoncés d'examen et de certification en échocardiographie en soins intensifs avancée.

Le paysage de la compétence et de la certification en échographie PoCUS ne peut pas être compris si l'on ne maîtrise pas complètement la nomenclature qui a été utilisée jusqu'à maintenant (Figure). Le terme générique d'échographie en soins intensifs (CCUS - critical care ultrasound) est le terme correct utilisé par les intensivistes et recouvre les deux principaux types d'échocardiographies en soins intensifs (CCE - critical care echocardiography) et d'échographies générales en soins intensifs (GCCUS general critical care ultrasound). ${ }^{1}$ L'échocardiographie en soins intensifs est elle-même divisée en groupes d'habiletés de base (BCCE) et avancées (ACCE).

Un praticien de niveau élémentaire doit être capable de générer cinq vues transthoraciques de base, interpréter la taille et la fonction globale des ventricules gauche et droit, identifier un épanchement péricardique, évaluer la veine cave inférieure et identifier une atteinte valvulaire catastrophique du côté gauche. La BCCE est tellement indispensable à la pratique moderne des soins intensifs qu'elle a été reconnue comme habileté essentielle pour tous les intensivistes par la communauté internationale des médecins de soins intensifs ${ }^{1,2}$ et bénéficie d'une base de données en expansion. ${ }^{3}$ Comme pour les autres habiletés essentielles des intensivistes (bronchoscopie, voie veineuse centrale, gestion des voies respiratoires), la BCCE (comme d'ailleurs les GCCUS) doit être enseignée sous la supervision d'un expert local au cours de la formation post doctorale (fellowship). Il est important de rappeler qu'une certification officielle pour les BCCE et les GCCUS n'a pas été jugée nécessaire, à la fois sur le plan international $^{1}$ et par un consensus unanime d'experts canadiens en CCUS, ${ }^{4}$ une position reconnue par la Société canadienne de soins intensifs en 2013.

Pour ce qui concerne les applications avancées de l'échocardiographie, on constate un démarcage très important entre la démarche et la philosophie entourant la formation, et la certification. Les applications avancées nécessitent des habiletés cognitives et procédurales plus importantes, sont plus quantitatives (et donc sujettes aux erreurs) et s'approchent davantage des examens échocardiographiques à visée diagnostique effectués par les cardiologues ou des examens peropératoires effectués par les anesthésiologistes cardiaques. Dans le champ d'application 
des ACCE, un intensiviste peut utiliser une approche transthoracique ou transœsophagienne pour répondre à des questions hémodynamiques (débit cardiaque, réponse au volume, ou interactions cœur-poumons), pour résoudre des problèmes diagnostiques (étiologie d'un choc, origine d'une embolie, endocardite ou important dysfonctionnement valvulaire) ou pour guider des procédures (insertion d'un stimulateur cardiaque ou canulation pour oxygénation extracorporelle). Compte tenu de cette sophistication inhérente et de la ressemblance avec une échocardiographie complète, la communauté internationale est d'accord pour estimer qu'une certification formelle est souhaitable. ${ }^{2,5}$ Bien que l'Amérique du Nord ne dispose pas (jusqu'à maintenant) de mécanisme pour répondre à cet appel en faveur d'une certification avancée, déjà vieux d'une décennie, d'autres pays ont réussi la mise en œuvre de telles initiatives, comme en témoigne le programme de diplôme européen en échocardiographie. ${ }^{6}$

Toutefois, à partir de 2019, un examen et une certification en échocardiographie avancée en soins intensifs deviendront une réalité en Amérique du Nord. Le National Board of Echocardiography (NBE), l'organisation supervisant la certification pour les échocardiographies globales et périopératoires, a constitué un partenariat avec de nombreuses sociétés de soins intensifs d'Amérique du Nord dans le but de créer un examen et une voie de certification pour les ACCE. Ce processus bénéficie d'un large soutien, notamment (parmi d'autres) : l'American College of Chest Physicians, la Society of Cardiovascular Anesthesiologists, la Society of Critical Care Medicine, l'American Thoracic Society, l'American Society of Echocardiography, et l'American College of Emergency Physicians. Bien que d'origine américaine, les examens du NBE sont utilisés depuis longtemps par d'autres groupes de spécialités au Canada et la valeur de cette certification aura les mêmes répercussions avec la création de cette filière.

Les détails sont encore en cours de finalisation, mais la filière du NBE pour les soins intensifs présentera de grandes similitudes avec le processus actuel de certification pour les cardiologues et anesthésiologistes cardiaques. La piste des soins intensifs comportera en particulier un examen standardisé sur les connaissances (avec un test d'évaluation avancée) et une formation pratique; les candidats devront apporter la preuve qu'ils ont rempli un portefeuille d'échographies, sous supervision locale. L'objectif de ce processus de certification est de légitimiser et de soutenir l'utilisation du groupe d'habiletés des ACCE par les intensivistes qui répondent aux normes élevées de formation cognitive et procédurale définies dans la déclaration du consensus international sur la formation aux ACCE. ${ }^{5}$
Lorsque l'on considère le modèle de certification pour les ACCE, l'exemple historique récent fourni par l'anesthésiologie cardiaque peut être instructif. Confrontée au défi similaire de l'adoption d'une compétence avancée à l'échographie en pratique quotidienne, la spécialité a adopté un modèle de filière de certification avancée comparable. Ce parcours a aidé à surmonter la difficile transition nécessaire pour obtenir une masse critique de praticiens avancés compétents, rappelant le climat actuel en soins intensifs par rapport aux ACCE. De la même manière que la certification en échocardiographie peropératoire avancée a constitué un jalon essentiel en anesthésiologie cardiaque, nous pensons que la certification en ACCE des intensivistes contribuera à créer une nouvelle ère dans la pratique des soins intensifs.

On s'attend à un intérêt important, ${ }^{7}$ mais il faut souligner que la nouvelle filière du NBE ne porte pas ou ne restreint pas l'utilisation régulière des applications échocardiographiques de base par ceux qui ont été correctement formés. Aucun obstacle, de quelque sorte que ce soit, ne doit empêcher l'utilisation des applications échographiques de base par les praticiens exerçant en soins intensifs. L'échocardiographie de base en soins intensifs (BCCE) et les GCCUS sont des compétences de la médecine de soins intensifs ${ }^{8}$ et leur utilisation doit continuer à s'épanouir, avec l'encadrement d'experts locaux conformément aux lignes directrices nationales ${ }^{4}$ et internationales. $^{2}$

Nous sommes favorables à cette nouvelle filière de certification pour les ACCE et nous encourageons tous les leaders en puissance en CCUS à l'obtenir. L'histoire des succès du NBE dans l'administration des examens d'échocardiographie couplée à un vaste soutien de la société conduira, à notre avis, à une adoption importante et à un accueil positif de la communauté nord-américaine des soins intensifs. En plus de certifier ceux qui sont compétents en ACCE et d'introduire officiellement l'échocardiographie avancée dans la pratique nord-américaine des soins intensifs, cette nouvelle filière aura deux effets supplémentaires qui profiteront globalement aux soins intensifs. Tout d'abord, ceux qui seront certifiés en ACCE pourront être des références et devenir des champions au niveau local, contribuant à combler l'insuffisance actuelle en supervision et formation dans le domaine des BCCE. Ensuite, une conséquence imprévue de la certification avancée est que les BCCE et GCCUS, laissées volontairement à l'écart de cette filière, seront d'autant plus reconnues comme des habiletés essentielles des intensivistes. En conséquence, les éducateurs et régulateurs des formations peuvent et doivent agir pour assurer leur plus grande dissémination. 
Conflicts of interest None declared.

Editorial responsibility This submission was handled by Dr. Gregory L. Bryson, Deputy Editor-in-Chief, Canadian Journal of Anesthesia.

Conflits d'intérêts Aucun déclaré.

Responsabilité éditoriale Cet article a été traité par le Dr Gregory L. Bryson, rédacteur en chef adjoint, Journal canadien d'anesthésie.

\section{References}

1. Mayo P, Beaulieu Y, Doelken P, et al. American College of Chest Physicians/La Société de Réanimation de Langue Française statement on competence in critical care ultrasonography. Chest 2009; 135: 1050-60.

2. Expert Round Table on Ultrasound in ICU. International expert statement on training standards for critical care ultrasonography. Intensive Care Med 2011; 37: 1077-83.
3. Levitov A, Frankel HL, Blaivas $M$, et al. Guidelines for the appropriate use of bedside general and cardiac ultrasonography in the evaluation of critically ill patients - part II: cardiac ulrasonography. Crit Care Med 2016; 44: 1206-27.

4. Arntfield RT, Millington S, Ainsworth $C$, et al. Canadian recommendations for critical care ultrasound training and competency. Can Respir J 2014; 21: 341-5.

5. Expert Round Table on Echocardiography in ICU. International consensus statement on training standards for advanced critical care echocardiography. Intensive Care Med 2014; 40: 654-66.

6. European Society of Intensive Care Medicine. European Diploma in Advanced Critical Care Echocardiography. Available from URL: https://www.esicm.org/education/edec-2 (accessed December 2017).

7. Mayo PH, Narasimhan M, Koenig S. Advanced critical care echocardiography: the intensivist as the ACCE of hearts. Chest 2017; 152: 4-5.

8. Millington SJ. Cardiac ultrasound is a competency of critical care medicine. Crit Care Med 2017; 45: 1555-7. 